\title{
LES DEICTIQUES DANS LES TEXTES JURIDIQUES
}

\section{INTRODUCTION}

Comme toute autre langue, la langue slovène se subdivise en plusieurs entités différant entre elles du point de vue social et fonctionnel. Y coexistent les sociolectes des domaines et métiers les plus divers. Certains d'entre eux se sont solidement ancrés au fil des années dans le système de la langue slovène, d'autres sont encore en cours de formation ou/et d'adaptation aux lois morphologiques, lexicales et syntaxiques de la langue slovène.

Dans la langue slovène, nous rencontrons des textes juridiques dès le $\mathrm{XVI}^{\mathrm{e}}$ siècle, notamment dans « Gorske bukve », le premier recueil de lois en slovène. En 1582, Andrej Recelj, un curé de Basse-Carniole, a traduit de l'allemand en slovène un recueil de lois styrien comprenant tous les textes régulant l'exploitation des vignobles, les routes, le nettoyage, etc. Ensuite, jusqu'au milieu du XIX ${ }^{\mathrm{e}}$ siècle, quand le Code national fut traduit sous la direction de Matej Cigale, nous pouvons observer le développement continu de la langue juridique slovène. Certes, il s'agit principalement de traductions de l'allemand, cependant il ne faut pas négliger le fait que, grâce à elles, les futurs juristes pouvaient s'instruire en slovène dès le milieu du XIX ${ }^{\mathrm{e}}$ siècle. L'essor des études juridiques slovène a été consacré en 1919 avec la fondation de la Faculté de droit de Ljubljana. Autant les traductions des textes juridiques que la possibilité de suivre une formation juridique dispensée en slovène stimulèrent l'essor d'une langue juridique slovène autonome.

\section{THÉORIE DES VARIÉTÉS FONCTIONNELLES DE LA LANGUE}

L'évolution d'une langue de spécialité déterminée n'est pas spécifique uniquement au niveau du lexique, c'est-à-dire de la terminologie. Pour mener pleinement sa propre vie, la langue de spécialité doit également se conformer à d'éventuelles particularités syntaxiques (par exemple, l'ordre des mots et la cohésion entre les différents éléments reliés entre eux).

Ainsi, en 1933, Božo Vodušek écrit :

Les grands principes d'une future stylistique slovène de ce type devraient consister à aboutir à la plus grande adéquation possible entre la langue écrite

Adresse de l'auteur : Filozofska fakulteta, Univerza v Ljubljani, Aškerčeva 2, 1000 Ljubljana, Slovénie. Courriel : mojca.smolej@ff.uni-lj.si 
et le slovène effectivement parlé par la population, à un principe de différenciation de la langue en fonction de groupes sociologiques déterminés et à la plus grande liberté possible laissée à chaque personne individuelle tant que cette dernière ne transgresse pas l'harmonie des lois historico-évolutives avérées de la langue slovène et ne dépasse pas les limites de toute expression linguistique normative (Vodušek $1933:$ 76).

Le principe de différenciation en fonction des groupes sociologiques est mentionné par Vodušek de manière très superficielle. En effet, pour lui, le principe fondamental reste l'adéquation entre la langue vivante, effectivement parlée par les locuteurs, et la langue standard écrite qui inclut également les langues de spécialité. Nous pouvons donc en déduire que la langue de spécialité, dans notre cas la langue juridique, doit, elle aussi, respecter les règles de la langue générale. La langue de spécialité ou langue juridique est une unité à l'intérieur d'une langue déterminée. Du fait qu'elle est fondée sur les règles de la langue générale, elle coïncide partiellement avec la langue générale ou, pour reprendre les mots de B. Vodušek, avec la langue effectivement parlée par la population. Notons qu'il est intéressant de constater qu'il peut y avoir entre les deux entités également des emprunts ou échanges réciproques. La langue de spécialité (ici, la langue juridique) ne fait pas qu'emprunter certains éléments à la langue générale ; on rencontre également dans la langue générale des éléments qui proviennent initialement de la langue des juristes. Bien sûr, on peut le plus souvent parler de déterminologisation où certaines composantes sémantiques d'un terme restent reconnaissables après le passage de la langue de spécialité dans la langue générale. Cependant, sur la base d'un transfert sémantique complet, peut aussi apparaître un sens tout à fait nouveau ne désignant plus le même concept que le terme d'origine. Le lexème sodišče (tribunal, cour de justice) est un exemple de déterminologisation partielle où seules certaines composantes sémantiques se sont perdues.

Toute langue de spécialité, y compris bien sûr la langue juridique, témoigne de caractéristiques propres qui n'apparaissent pas seulement au niveau lexical, mais aussi aux autres niveaux (notamment syntaxique et textuel). C'est ce que fait remarquer Rudolf Kolarič dès 1956 dans son article Leposlovni jeziki in strokovni jeziki (Les langues littéraires et les langues professionnelles).

Chaque domaine de spécialité a, en dehors de sa terminologie purement technique, également sa propre manière d'agencer et de construire les phrases, son propre usage des adjectifs et autres mots, ses dictons spécifiques et même sa propre manière d'utiliser la ponctuation. Indépendamment des termes techniques différents qu'il emploie, un médecin s'exprime tout à fait différemment, par exemple, d'un ingénieur en génie mécanique ou en génie électrique. Même un physicien, un ingénieur en génie mécanique et un ingénieur en génie électrique, dont les domaines sont pourtant assez proches, se distinguent entre eux par un mode d'expression qui leur est propre même lorsqu'ils parlent du même sujet technique (Kolarič 1956 : 118). 
Dans le présent article, nous nous intéresserons avant tout au niveau syntaxique, plus exactement au niveau de la cohésion qui est fondée, entre autres, sur l'usage de moyens déictiques très divers. Nous nous concentrerons sur la question de savoir si l'usage des déictiques dans les textes juridiques est adéquat ou, autrement dit, quand cet usage transgresse les lois générales de la langue juridique.

Auparavant, nous tenterons d'énoncer les caractéristiques fondamentales des langues de spécialité, notamment de la langue juridique, à l'aide de la théorie fonctionnelle élaborée dès 1963 par Havranek ainsi que la théorie des variétés fonctionnelles de la langue énoncée d'un point de vue pragmatique par A. Skubic. Ce faisant, nous aurons également recours aux théories de Nikola Visković (Jezik prava, 1989) et de Matthias Klatt (Making the law explicit, 2008).

N. Visković applique à la langue juridique les affirmations que R. Kolarič avait précédemment formulées concernant les langues de spécialité en général.

La langue juridique ne peut être tout à fait autonome par aucune de ses dimensions linguistiques. C'est nécessairement un sous-système de la langue générale à laquelle elle doit la majeure partie de ses caractéristiques formelles et significatives. Cependant, ce sous-système linguistique est un sociolecte spécialisé et technique qui se différencie en bien des traits de la langue générale standard et des autres sous-systèmes linguistiques. Les particularités de la langue juridique apparaissent à tous les niveaux de l'activité linguistique. [...]. Nous verrons dans quelle mesure il est erroné d'affirmer que le droit n'est pas doté de niveau syntaxique propre (Viskovič 1989 : 25-26).

De même, M. Klatt (2008 : 181-207) souligne à plusieurs reprises l'importance de la vérification constante de la signification grammaticale/linguistique fondée sur l'examen de tous les niveaux linguistiques, pas seulement au niveau sémantique (seul le niveau phonétique peut conditionnellement être exclu). Ainsi, la signification linguistique découlant du socle propositionnel devrait être libérée de toutes éventuelles interprétations subjectives. Malheureusement, le doute apparaît aussi à ce niveau. C'est ce que montre, notamment, l'exemple mentionné par Olga Kunst-Gnamuš dans son article « Težave upovedovalne teorije in smeri njenega razvoja » (« Les difficultés de la théorie de l'énonciation et les orientations de son évolution »).

Bien que nous connaissions les mots employés et que nous maîtrisions l'analyse syntaxique en éléments phrastiques, nous ne savons pas expliquer la signification de la phrase Pozdravljanje naših brigadirjev je veselo (Les saluts de nos brigadiers sont joyeux). Qu'est-ce qui est l'objet du récit? Je veselo (Est joyeux). Qu'est-ce qui est joyeux ? Pozdravljanje naših brigadirjev (Les saluts de nos brigadiers) ? L'analyse de la structure de surface ne permet pas de répondre aux principales questions : qui accomplit l'acte, qui est celui qui salue ? Nous avons affaire à un rapport mettant en présence une expression et deux sens [...]. À côté de la structure de surface se dévoile 
une autre structure porteuse d'une nouvelle signification : l'expression de surface et la signification ne coïncident pas. Au contraire, l'expression est un moyen permettant de flouter la signification. Elle ne le fait pas toujours et pas nécessairement, mais la possibilité existe et peut être utilisée (KunstGnamuš 1987/88: 8).

Pour poser une définition générale de la langue de spécialité et, donc, de la langue juridique, nous nous appuierons sur les théories fonctionnelles de B. Havranek (Studie o spisovnem jazyce, 1963) et d'A. Skubic (Klasifikacija funkcijske zvrstnosti in pragmatična definicija jezika, 1994/95).

Les points de départ des deux théories sont contradictoires, la première partant du structuralisme et la seconde de la pragmatique. Cependant, en dépit de la distance temporelle et théorique qui les sépare, ces deux théories se complètent l'une l'autre du fait qu'elles tendent vers le même objectif : déterminer les fonctions et les propriétés de chaque sociolectes. Dans notre cas, nous limiterons notre analyse à la langue de spécialité, plus précisément la langue juridique.

Havranek est l'un des premiers à avoir posé le critère sur trois niveaux permettant l'étude fonctionnelle des textes. Il les étudie en fonction a) de l'unicité ou la disparité du niveau sémantique, $b$ ) de la relation libre ou conventionnelle au message et c) de l'achèvement ou du non achèvement du texte (Pogorelec $1986: 11-22$ ).

Skubic (1994/95 : 163-165) classe les textes juridiques dans la catégorie du discours performatif conventionnel caractérisé par une structuration parfaite du texte en ses différents éléments formels et sémantiques ainsi que par une nette impersonnalité. Par ailleurs, les textes performatifs conventionnels doivent être extrêmement précis et dénués de toute ambiguité.

Du fait qu'ils interviennent de manière déterminante dans la vie des gens, dans la gestion de leurs biens, etc., les textes performatifs conventionnels doivent être des conventions extrêmement précises et dénuées de toute ambiguité. Cela est typique dans les passages se rapportant constamment à des alinéas et points clairement déterminés, lors de répétitions constantes de mots ou syntagmes de référence (les déictiques et reprises abrégées sont généralement jugés indésirables en raison de leur ambiguïté potentielle : seules certaines expressions clés peuvent être abrégées à condition que cela soit explicitement mentionné [...] (Skubic 1994/95 : 164).

Le plus important pour la présente contribution est avant tout la partie de la citation mentionnant le caractère " indésirable " des moyens déictiques. En effet, ces derniers peuvent provoquer un rapport au message plus libre. Dans les pages suivantes, nous vérifierons le bien-fondé de cette affirmation à l'aide de textes juridiques concrets.

Pour résumer ce qui vient d'être dit, nous pouvons écrire que les textes juridiques sont, par définition : 
- impersonnels (cela implique surtout l'exclusion totale de toute expressivité) ;

- clairement subdivisés (cela implique qu'ils sont élaborés avec précision et sont conformes à la structure textuelle prescrite) ;

- explicites et ;

- dénués d'ambiguité (cela implique qu'ils sont univoques et excluent totalement l'établissement d'une quelconque relation libre par rapport au contenu).

\section{LES DÉICTIQUES, ÉLÉMENTS PORTEURS D‘AMBIGUÏTÉ ?}

Selon J. Verschuern (2000 : 36-41), les déictiques sont des expressions impossibles à interpréter hors de leur contexte. Lors de l'élimination des éléments conditionnant le discours, qu'il soit écrit ou parlé, l'interprétation des déictiques n'est qu'apparente ou de l'ordre du « probable »; elle n'est absolument pas la seule possible. J. Verschueren classe les déictiques en quatre groupes : 1) les déictiques temporels, 2) les déictiques spatiaux, 3) les déictiques sociaux et 4) les déictiques discursifs. Par ailleurs, à l'intérieur de la déicticité, il faut distinguer les concepts relatif et absolu. Les déictiques relatifs sont des expressions sans référence générale (par exemple, ici, là, aujourd'hui, alors, etc.) et dont la signification à l'intérieur du centre déictique varie selon le locuteur ; au contraire, les déictiques absolus se caractérisent pas l'invariabilité de leur contenu sémantique quel que soit le centre déictique (par exemple, les quatre points cardinaux).

La propriété fondamentale des déictiques est donc de ne revêtir un sens dénué de toute ambiguïté qu'une fois replacés dans leur contexte. Pour définir avec précision le sens d'un déictique donné, il faut prendre en compte tous les aspects possibles du centre déictique. D'autre part, il faut nécessairement s'arrêter sur toutes les interprétations syntaxiques possibles du texte (de l'unité textuelle) où le déictique est utilisé.

En effet, les déictiques sont aussi des moyens cohésifs puissants qui sont souvent utilisés comme moyens anaphoriques qui remplacent dans le texte une expression coréférante utilisée précédemment dans un sens clairement déterminé. Les déictiques peuvent également être utilisés comme moyens cataphoriques utilisés dans le discours avant l'expression co-référante. Le plus souvent, la forme cataphorique s'utilise pour créer l'indécision ou pour attiser l'intérêt du récepteur. Ce qui est certain, c'est que, contrairement à l'usage de la forme anaphorique, l'usage de la forme cataphorique est inattendu dans les textes de spécialité, notamment dans les textes juridiques.

Dans les pages suivantes, nous nous concentrerons sur un exemple d'utilisation des déictiques comme moyen anaphorique et sur ses interprétations sémantiques possibles.

\subsection{Une loi prétendument ambiguë}

Les lois sont censées être explicites et dépourvues d'ambiguïté. Malheureusement, en raison de la nature même du domaine juridique, fondé sur l'utilisation de la langue que nous nous « approprions » nous aussi, les autres locuteurs, et en raison du fait que de nombreux facteurs sociaux (idiomatiques, psycho-physiques, élocutoires, etc.) influent sur l'utilisation et la compréhension de la langue, l'objectif donné peut rester inaccompli, le plus souvent involontairement et même sans que l'auteur en ait conscience. 
Nous concentrerons notre attention sur le deuxième alinéa de l'article 18 de la Loi sur les contentieux administratifs (ZUS-1), ${ }^{1}$ adoptée par l'Assemblée nationale de la République de Slovénie le 28 septembre 2006 et promulguée le 6 octobre de la même année :

Zastopnik javnega interesa lahko vloži tožbo na podlagi pooblastila vlade, kadar ta ugotovi, da je bil z upravnim aktom kršen zakon v škodo javnega interesa (Le représentant de l'intérêt public peut déposer plainte sur la base d'une procuration du gouvernement dans les cas où il constate que l'acte administratif transgresse la loi aux dépens de l'intérêt général).

Nous nous arrêterons sur cette loi dont l'interprétation a par le passé suscité de nombreuses difficultés et polémiques.

Pour mieux comprendre comment et pourquoi des interprétations équivoques sont apparues, il convient de décrire brièvement l'exemple concret qui a conduit à des interprétations contradictoires de l'article 18 de la Loi sur les contentieux administratifs déjà mentionné.

Le 2 septembre 2009, le Tribunal administratif ${ }^{2}$ a rejeté la plainte du procureur de la République contre la décision du Ministère de l'agriculture par laquelle le propriétaire $\mathrm{XY}$ se voyait restituer les trois chiens qui lui avaient été confisqués le 15 mars 2006 à la suite de l'agression dont avait été victime un homme de 36, gravement mordu par ces chiens. Le 22 juin 2009, le Ministère de l'agriculture a, après une série de procédures et en accord avec le jugement rendu par le Tribunal administratif, rendu une décision restituant les chiens à leur propriétaire. Le 23 juillet 2009, suite à la restitution de chiens, le procureur de la République dépose une plainte à l'encontre du Ministère de l'agriculture. Le 2 septembre 2009, le Tribunal administratif rejette cette plainte en invoquant le fait que le procureur de la République ne disposait pas d'une procuration du gouvernement pour effectuer cette démarche. Le 2 février 2010, les chiens restitués attaquent et tuent leur maître.

\subsubsection{Les deux bullmastiffs ont été sauvés par le pronom " ta"}

Le titre de cette section représente le titre d'un article publié dans le quotidien Dnevnik le 22 septembre 2009. ${ }^{3}$ Comme nous l'avons expliqué précédemment, le procureur de la République a déposé un recours auprès du tribunal administratif à l'encontre du Ministère de l'agriculture qui venait de restituer à leur propriétaire des chiens que le procureur de la République jugeait dangereux. En dépit de son bien-fondé, la plainte a été rejetée par le tribunal administratif. Le rejet repose uniquement sur le fait que le

1 http://www.uradni-list.si/1/objava.jsp?urlid=2006105\&stevilka=4487

2 Voir les sources suivantes : http://www.dnevnik.si/slovenija/v-ospredju/1042300929 ; http:// www.dnevnik.si/slovenija/v-ospredju/1042342800 ; http://www.delo.si/novice/kronika/aferabulmastifi-tozilka-zdaj-preganja-sodnico-in-novinarje.html ; http://www.delo.si/assets/media/ other/20110131//0130_AferaBaricevic.pdf

3 http://www.dnevnik.si/slovenija/v-ospredju/1042300929 
procureur de la République n'avait pas été mandaté par le gouvernement pour effectuer la démarche, ce qui signifie que le tribunal administratif n'a pas compris l'article 18 de la Loi sur les contentieux administratifs de la même manière que le procureur de la République. Le tribunal administratif a compris ${ }^{4}$ le pronom $t a$ comme une forme anaphorique se rapportant au substantif vlada situé dans la proposition principale (zastopnik javnega interesa lahko vloži tožbo na podlagi vlade, kadar ta ugotovi ...), tandis que le procureur de la République, dans sa contestation du rejet dont sa plainte a fait l'objet, se défend en invoquant la possibilité d'une autre interprétation du texte de loi. ${ }^{5}$ Selon lui, le pronom $t a$ se rapporte à la locution substantivale zastopnik javnega interesa (zastopnik javnega interesa lahko vloži tožbo na podlagi vlade, kadar ta ugotovi ...). Il est indéniable que ces deux interprétations sémantiques diffèrent beaucoup l'une de l'autre. D'après la deuxième interprétation, où le pronom $t a$ se rapporte au syntagme zastopnika javnega interesa (le représentant de l'intérêt général), c'est précisément ce dernier qui assume le rôle d'actant primaire dans la phrase subordonnée (kadar ta ugotovi, da je bil z upravnim aktom kršsen zakon $v$ škodo javnega interesa), tandis que le substantif vlada (le gouvernement) est totalement exclu du spectre des actants du socle sémantique de la subordonnée temporelle. Au contraire, d'après la première interprétation, le rôle d'actant primaire dans le socle sémantique de la subordonnée temporelle revient au substantif vlada (le gouvernement).

Le procureur de la République s'est donc fondé sur la seconde interprétation sémantique et, de ce fait, a déposé sa plainte auprès du tribunal administratif sans procuration en cours de validité délivrée par le gouvernement. En effet, il était d'avis que c'était à lui-même et non au gouvernement que revenait la tâche de déterminer si tel ou tel acte individuel enfreignait ou non la loi aux dépens de l'intérêt général. D'après cette seconde interprétation, le procureur de la République devait donc disposer uniquement d'une procuration générale du gouvernement et non d'une procuration portant sur chaque acte individuel. Or, le procureur de la République a obtenu une procuration générale du gouvernement dès le 5 avril $2007 .{ }^{6}$

En se fondant sur la première interprétation sémantique présentée, qui exige que le gouvernement rédige une procuration dans chaque cas particulier, le Tribunal administratif a rejeté la plainte.

Dans la suite du présent article, nous allons essayer de déterminer si la loi présentée est réellement ambiguë.

\subsubsection{Pourquoi la loi concernée n'est pas ambiguë}

Le deuxième alinéa de la loi concernée (ZUS-1) est constitué de trois propositions : une proposition principale (zastopnik javnega interesa lahko vloži tožbo na podlagi pooblastila vlade) qui, en tant que noyau de la phrase, exprime le rôle déclaratif, et deux propositions subordonnées (kadar ta ugotovi, da je bil z upravnim aktom kršen

$4 \quad$ Voir notes 2 et 3.

5 Voir notes 2 et 3.

6 Voir notes 2 et 3. 
zakon $v$ škodo javnega interesa) qui complètent le contenu exprimé par le noyau (elles remplissent la fonction de propositions circonstancielles de temps). En dehors de son rôle de complément temporel signalé déjà par la conjonction kadar (à chaque fois), la subordonnée de premier degré (kadar ta ugotovi) assume aussi, au niveau de la cohésion, un rôle de reprise avec l'enclitique $t a$. L'expression co-référante du pronom $t a$ utilisé anaphoriquement se trouve donc dans la proposition principale. Comme le pronom étudié appartient à la catégorie des substantifs, nous pouvons supposer en dehors de tout contexte qu'il se rapporte à un substantif précis, doté de sa signification pleine et entière dans la proposition principale. Pour déterminer le vrai co-référant, il convient d'opérer une analyse du socle sémantique de la proposition principale. Au niveau du socle sémantique, la proposition principale comporte trois participants : l'agent de l'action (zastopnik javnega interesa), l'objet (tožbo) et le complément circonstanciel de condition (na podlagi pooblastila vlade). Ce dernier comporte à son tour un agent de l'action implicite (vlada). Dans la proposition principale sont donc exprimés deux agents d'action, l'un explicite (zastopnik) et l'autre explicite (vlada). Par son contenu, l'agent implicite élargit le socle sémantique de la proposition principale, mais se retrouve au niveau secondaire de la proposition. En effet, en comparaison avec l'agent de l'action explicite, il a perdu tout rôle actif. L'étude de cette perte de rôle active est cruciale pour la compréhension de l'exemple auquel nous nous intéressons ici.

En effet, quand l'agent implicite ou secondaire de l'action clairement exprimé dans la proposition principale prend le rôle d'agent actif et explicite de l'acte dans la proposition subordonnée, la conversion doit être clairement indiquée, et ce uniquement avec des moyens linguistiques bien déterminés (par exemple, à l'aide de pronoms démonstratifs). Si le passage d'une catégorie à l'autre n'est pas clairement indiqué, on tiendra la conversion comme non réalisée, ce qui signifie que l'agent secondaire et implicite de l'action de la proposition principale conserve son rôle secondaire aussi dans la proposition subordonnée.

Nous tenterons d'illustrer ce que nous venons d'écrire en comparant l'article 18 de la Loi sur les contentieux administratifs (exemple 1) et le texte de ce même article dont nous aurons supprimé le pronom ta, prétendument ambigu (exemple 2).

1) Zastopnik javnega interesa lahko vloži tožbo na podlagi pooblastila vlade, kadar ta ugotovi, da je bil z upravnim aktom kršen zakon v škodo javnega interesa.

2) Zastopnik javnega interesa lahko vloži tožbo na podlagi pooblastila vlade, kadar ugotovi, da je bil z upravnim aktom kršen zakon v škodo javnega interesa. ${ }^{7}$

Dans l'exemple 2, l'agent explicite et central de l'action, zastopnik javnega interesa (le représentant de l'intérêt général) a conservé son rôle sémantique également dans la proposition temporelle. Cela apparaît surtout à travers le sujet au niveau structurel, exprimé à l'aide d'un morphème (ugotovi). En slovène, l'agent central de l'action de la proposition principale conserve son rôle le plus souvent à l'aide de désinences verbales à valeur anaphorique. En raison de l'emploi susmentionné, l'exemple 2 est incontestablement dénué de toute ambiguité. En effet, si l'auteur du texte original de l'article 18

7 En français, l'ambiguïté serait levée par l'utilisation des pronoms « celui-ci » et « celui-là » dont le slovène ne possède pas d'équivalent. 
de la Loi sur les contentieux administratifs avait voulu exprimer que l'agent de l'action était le même tant dans la proposition principale que dans la proposition subordonnée, il se serait contenté, en accord avec les règles de la langue slovène, d'utiliser des désinences verbales à valeur anaphorique.

Dans l'exemple 1, la subordonnée temporelle comprend la forme $t a$, moyen anaphorique causant la conversion de l'agent implicite de l'action présentée dans la proposition principale (vlada). En raison de l'utilisation du pronom $t a$, il apparait clairement que le rôle d'agent primaire de l'action n'est plus assuré par le sujet de la proposition principale zastopnik javnega interesa, mais par le sujet psychologique qui participe du complément circonstanciel de conséquence (vlada).

Il s'ensuit donc que l'article 18 de la Loi sur les contentieux administratifs n'est pas ambigu L'ambiguïté qui lui a été reprochée est due à une mauvaise compréhension ou à une méconnaissance, volontaire ou non, des rôles sémantico-syntaxiques et de l'usage des formes anaphoriques, ce qui est la conséquence d'une lecture du texte limitée au niveau de surface, le niveau plus profond ayant été totalement exclu.

Dans l'article 18 de la Loi sur les contentieux administratifs, le pronom ta se rapporte donc incontestablement au substantif vlada (na podlagi pooblastila vlade). Le tribunal a donc tout à fait légitimement débouté de sa plainte l'avocat général qui ne disposait d'aucune procuration valide du gouvernement.

\section{CONCLUSION}

Pour finir, revenons au point de départ de la théorie des variétés fonctionnelles de la langue affirmant que l'emploi des déictiques dans les textes juridiques doit être évité. Demandons-nous donc à nouveau si cette assertion est fondée. La réponse ne peut en aucun cas être affirmative. Bien que les déictiques ne puissent pas être interprétés en dehors de leur contexte, leur usage est attendu. Comme nous l'avons constaté en étudiant un exemple concret, l'interprétation du sens est le plus souvent liée aux lois linguistico-grammaticales codées dans le système même de la langue et qui, de ce fait, imposent une compréhension univoque du message textuel. Avec l'usage des déictiques, nous évitons la saturation textuelle résultant souvent d'une répétition explicite des termes excessive (Pogorelec 1989: $35,36,41)$. Or, toute saturation textuelle transgresse et bouleverse les principes fondamentaux de la construction textuelle, y compris ceux des textes juridiques. Les critères voulant que tout texte juridique soit élaboré de façon à être à la fois explicite et univoque peuvent donc être respectés même dans le cas d'une utilisation de formes anaphoriques.

Comme nous l'avons écrit précédemment, toute élaboration d'un texte, en particulier s'agissant d'un texte juridique, devrait inclure une phase de relecture finale qui devrait être effectuée tant au niveau des structures de surface qu'au niveau des structures plus profondes du texte. L'exclusion de l'un des niveaux revient à exclure les critères fondamentaux régissant la textualité des textes juridiques, ce qui peut, bien sûr, conduire par la suite à des interprétations sémantiques ambiguës. 


\section{Bibliographie}

\section{Sources primaires}

Afera bulmastifi : tožilka zdaj preganja sodnico in novinarje. 31 mai 2013. http://www.delo. si/novice/kronika/afera-bulmastifi-tozilka-zdaj-preganja-sodnico-in-novinarje.html

Kronologija afere bulmastifi. 31 mai 2013. http://www.delo.si/assets/media/ other/20110131//0130_AferaBaricevic.pdf

Nevarna bulmastifa je rešil zaimek ta. 31 mai 2013. http://www.dnevnik.si/ slovenija/v-ospredju/1042300929

Nevarni bulmastif Atlas dobil vzrejno dovoljenje. 31 mai 2013. http://www.dnevnik.si/ slovenija/v-ospredju/1042342800

Zakon o upravnem sporu. 31 mai 2013. http://www.uradni-list.si/1/objava. jsp?urlid $=2006105 \&$ stevilka $=4487$

\section{Références}

APOVNIK, Pavel/Aleksander FERI/Karlo PRIMOŽIČ (1999) Slovenski pravni leksikon z nemškimi in italijanskimi ustreznicami geselskih besed. Ljubljana : OSTsvetovalne storitve.

FERBEŽAR, Ina/Marko STABEJ (2009) « Razumljivost v slovenski pravni normi. » In : M. Stabej (ed.), Infrastruktura slovenščine in slovenistike. Obdobja 28. Ljubljana : ZZFF, 137-143.

GIBBONS, John (ed.) (1994) Language and the law. London : Longman.

KLATT, Matthias (2008) Making the law explicit. The normativity of legal argumentation. Oxford : Hart Publishing.

KOLARIČ, Rudolf (1956) « Leposlovni jezik in strokovni jeziki. » Jezik in slovstvo 2/3, 425-437.

KUNST-GNAMUŠ, Olga (1987/88) « Težave upovedovalne teorije in smeri njenega razvoja. » Jezik in slovstvo 33/1-2, 8-16.

POGORELEC, Breda (1986) «Znanstveno besedilo, njegove jezikoslovne prvine in slog. » In : A.Vidovič Muha (ed.), Slovenski jezikv znanosti 1. Ljubljana : ZIFF, 11-22.

POGORELEC, Breda (1989) «Slovenska pravna besedila : vprašanja jezika in sloga pri oblikovanju besedil. » In : A. Vidovič-Muha/N. Šumi (eds), Slovenski jezik v znanosti 2. Ljubljana : ZIFF, 35-43.

SKUBIC, Andrej (1994/95) « Klasifikacija funkcijske zvrstnosti in pragmatična definicija funkcije. » Jezik in slovstvo 15/5, 155-168.

VERSCHUEREN, Jef (2000) Razumeti pragmatiko. Ljubljana : *cf.

VISKOVIĆ, Nikola (1989) Jezik prava. Zagreb : ITRO »NAPRIJED« OOUR.

VODUŠEK, Božo (1933) « Za preureditev nazora o jeziku. » In : R. Ložar (ed.), Krog - zbornik umetnosti in razprav. Ljubljana : s.n., 66-76.

ŽAGAR, Mojca (2007) « Determinologizacija v splošnih in terminoloških slovarjih. » In : I. Orel (ed.), Razvoj slovenskega strokovnega jezika. Obdobja 24. Ljubljana : Center za slovenščino kot drugi/tuji jezik pri Oddelku za slovenistiko Filozofske fakultete UL, 599-609. 


\section{Résumé \\ LES DEICTIQUES DANS LES TEXTES JURIDIQUES}

Par définition, la langue juridique devrait être univoque, explicite et précise. Les moyens linguistiques, tant lexicaux que structurels, sont censés instaurer une relation de cause à effet optimale entre la pensée et son expression. Ainsi, le rédacteur des textes juridiques a le devoir de vérifier scrupuleusement l'adéquation de la pensée ou du contenu avec les moyens linguistiques utilisés; par ailleurs, au niveau structurel (par exemple, de l'ordre des mots), il doit effectuer des vérifications et éliminer toute éventuelle ambiguïté ou imprécision.

Nous prendrons pour point de départ de notre analyse des textes juridiques en vigueur transgressant d'une manière ou d'une autre la règle de clarté et de précision. L'emploi des déictiques est l'une des fréquentes causes d'ambiguïté. Le deuxième alinéa de l'article 18 de la Loi sur les contentieux administratifs (ZUS-1) présente un bon exemple de ce type d'emploi. Ainsi, nous lisons que « zastopnik javnega interesa lahko vloži tožbo na podlagi pooblastila vlade, kadar ta ugotovi, da je bil z upravnim aktom kršen zakon $v$ škodo javnega interesa » (« le représentant de l'intérêt public peut déposer plainte sur la base d'une procuration du gouvernement dans les cas où il constate que l'acte administratif transgresse la loi aux dépens de l'intérêt général »). Le pronom « ta », employé de manière anaphorique dans la proposition subordonnée, peut se référer à deux entités distinctes, « le représentant de l'intérêt public » (« zastopnik javnega interesa ») et « le gouvernement» (« vlada »), ce qui permet une interprétation abusive de la loi. En 2010, cette ambiguité a même indirectement conduit à la mort de l'un de ceux qui s'en était servi pour contourner la loi.

Mots-clés : texte juridique, déictiques, ambiguïté, structure profonde.

\section{Povzetek \\ DEIKTI IN PRAVNA BESEDILA}

Pravni jezik naj bi bil po definiciji nedvoumen, ekspliciten in natančen. Jezikovna sredstva, tako besedna kot strukturna, naj bi optimalno vzpostavljala vzročnoposledično razmerje med mislijo in njeno ubeseditvijo. Na ravni besede je pisec pravnih besedil tako dolžan dosledno preverjati prekrivnost miselne ali vsebinske podstave $\mathrm{z}$ jezikovnim sredstvom, na ravni strukture (npr. besedni red) pa mora preveriti in posledično odstraniti kakršnokoli možno vnašanje nedvoumnosti oz. neeksplicitnosti.

Izhodišče analize predstavlja 2. odstavek 18. člena Zakona o upravnem sporu. »Zastopnik javnega interesa lahko vloži tožbo na podlagi pooblastila vlade, kadar ta ugotovi, da je bil z upravnim aktom kršen zakon v škodo javnega interesa « (2. odstavek 18. člena ZUS-1).

Anaforično rabljeni zaimek »ta« $\mathrm{v}$ nadrednem odvisniku se lahko nanaša na dva različna koreferirajoča izraza. Omenjena nejasnost oz., natančneje, raba zaimka »ta« omogoča legitimno zlorabo zakonodaje, leta 2010 pa je botrovala tudi hudi človeški tragediji (smrti).

Ključne besede: pravna besedila, deikti, dvoumnost, propozicijska skladnja. 